\title{
Stilbene Glucoside, a Putative Sleep Promoting Constituent from Polygonum multiflorum Affects Sleep Homeostasis by Affecting the Activities of Lactate Dehydrogenase and Salivary Alpha Amylase
}

\author{
Qian Wei, ${ }^{a}$ Guang Ta, ${ }^{b}$ Wenjing He, ${ }^{b}$ Wei Wang, ${ }^{b}$ and Qiucheng $\mathrm{Wu}^{*, b}$ \\ ${ }^{a}$ Heart Disease Diagnosis and Treatment Center, The Affiliated Hospital to Changchun University of Chinese \\ Medicine; Changchun 130021, China: and ${ }^{b}$ Department of Emergency and ICU, The Affiliated Hospital to Changchun \\ University of Chinese Medicine; No.1478, Gongnong Road, Chaoyang District, Changchun 130021, China. \\ Received March 29, 2017; accepted August 8, 2017
}

\begin{abstract}
Chinese herbal medicine (CHM) has been used for treating insomnia for centuries. The most used CHM for insomnia was Polygonum multiflorum. However, the molecular mechanism for CHM preventing insomnia is unknown. Stilbene glucoside (THSG), an important active component of $\boldsymbol{P}$. multiflorum, may play an important role for treating insomnia. To test the hypothesis, Kunming mice were treated with different dosages of THSG. To examine the sleep duration, a computer-controlled sleep-wake detection system was implemented. Electroencephalogram (EEG) and electromyogram (EMG) electrodes were implanted to determine sleep-wake state. RT-PCR and Western blot was used to measure the levels of lactate dehydrogenase (LDH) and saliva alpha amylase. Spearman's rank correlation coefficient was used to identify the strength of correlation between the variables. The results showed that THSG significantly prolonged the sleep time of the mice $(p<0.01)$. THSG changed sleep profile by reducing wake and rapid eye movement (REM) period, and increasing non-REM period. RT-PCR and Western blot analysis showed that THSG could down-regulate the levels of $\mathbf{L D H}$ and saliva alpha amylase $(p<0.05)$. The level of lactate and glucose was positively related with the activity of $\mathrm{LDH}$ and saliva alpha amylase $(p<0.05)$, respectively. On the other hand, the activities of LDH and amylase were negatively associated with sleep duration $(\boldsymbol{p}<\mathbf{0 . 0 5})$. The levels of lactate and glucose affect sleep homeostasis. Thus, THSG may prevent insomnia by regulating sleep duration via LDH and salivary alpha amylase.
\end{abstract}

Key words stilbene glucoside; Polygonum multiflorum; lactate dehydrogenase; amylase; sleep; mouse

Insomnia has been recognized as a major public health issue. ${ }^{1,2)}$ Insomnia is associated with a high risk for hypertension or deficits in neuropsychological performance. ${ }^{3,4)}$ Thus it is urgent to explore the medicine that can improve the sleep quality. Chinese herbal medicine (CHM) has been used for treating sleep disorder in Asian for centuries. CHM is safe and efficient in the clinical treatment of insomnia with little side effects. Furthermore, the development of a new sedative hypnotic drugs becomes possible by screening the active ingredients of CHM. ${ }^{5)}$ The mostly used CHM for treating insomnia is Polygonum multiflorum. ${ }^{6}$ ) Specifically, P. multiflorum is the source of two different herbal medicine materials. One is the root, called heshouwu and the other is the stem (in the form of a vine), called yejiaoteng. Different plant parts have different levels of constituents affecting their pharmacology, clinical applications, and dosages. CHM practitioners also recommend the root and stems for different uses and the root is very rarely used for treating insomnia. However, one main active component from the root of $P$. multiflorum is stilbene glucoside (THSG, 2,3,5,4'-tetrahydroxystilbene-2-O-beta-D-glucoside), ${ }^{7)}$ which can offer the potential to prevent and treat Alzheimer's disease. ${ }^{8)}$ THSG has also been reported to improve the learning and memory of aged rats. ${ }^{9)}$ Whether THSG can improve the sleep quality, such as prolonging the sleep time, is still unknown. Meanwhile, we also want to know the molecular mechanism if THSG can increase the sleep time.

Recently, salivary alpha amylase (Fig. 1A) has been reported to be highly correlated with sleep drive. ${ }^{10)}$ The final products of amylase activity are glucose (Fig. 1B), which is also associated with sleep situation. ${ }^{11)}$ More importantly, salivary alpha amylase is also responsive to extended waking. On the other hand, it is well established that brain metabolism is higher during wake and rapid eye movement (REM) sleep than that in nonrapid eye movement (NREM). Most of the brain's energy is used to maintain neuronal firing and glutamatergic transmission. ${ }^{12}$ The increased release of oxytocin during lactation has been shown to be dependent upon glutamatergic transmission and is associated with an increased synaptic innervation of the supraoptic nucleus. ${ }^{13)}$ Extracellular lactate concentration is associated with survival and neurologic outcome in patients with cardiac arrest ${ }^{14)}$ and another reliable sleep component. ${ }^{15)}$ Rapid and sustained increases in cortical lactate concentration can be immediately observed during waking and REM sleep. Elevated lactate concentration is also maintained throughout a period of continuous waking. ${ }^{15)}$ A sustained decline in lactate concentration was measured during NREM sleep. The similar results were reported in other paper: lactate progressively increases during waking and REM sleep and decreases during NREM sleep. The observed state-dependent changes in lactate are consistent with higher brain metabolism during waking and REM sleep in comparison with that in NREM sleep. ${ }^{16)}$ On the other hand, the concentration of lactate (Fig. 1D) is determined by lactate dehydrogenase (LDH) (Fig. 1C). ${ }^{17)}$ Thus, LDH will be a potential biomarker for studying sleep problems.

From above results, we conjecture that the function of $P$. multiflorum preventing insomnia may be related with THSG while THSG may affect the protein levels of the sleep homeo- 
A

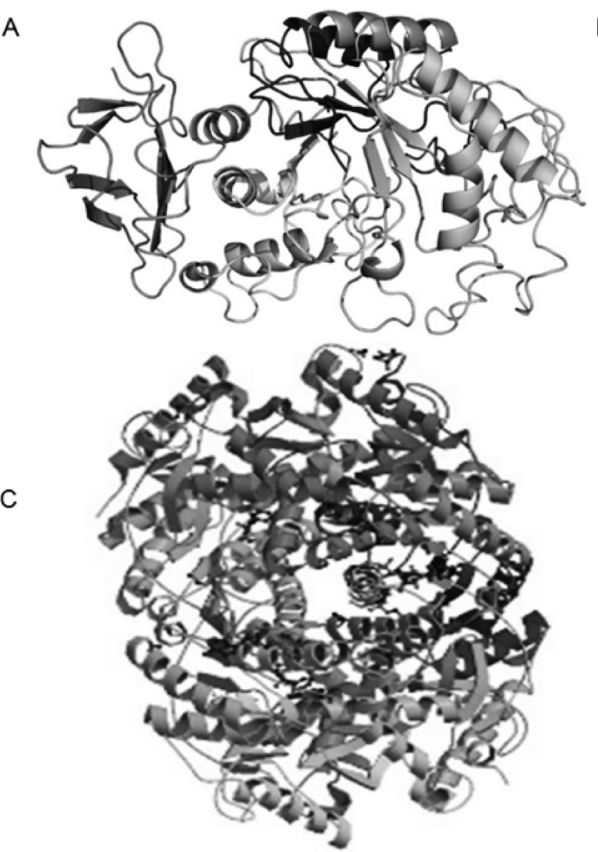

B<smiles>COC1C(O)C(O)C(O)C(O)C1CO</smiles>

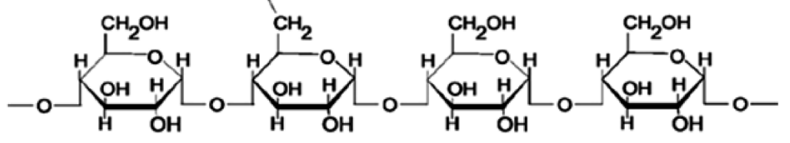

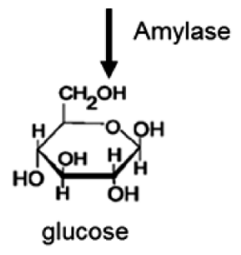

D

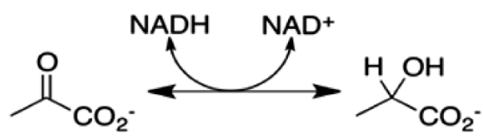

pyruvate

Fig. 1. The Catalyzing Functions of Salivary Alpha Amylase and Lactate Dehydrogenase

(A) The crystal structure of salivary alpha amylase. (B) Alpha amylase cleavages alpha bonds of alpha-linked polysaccharides of starch and glycogen, and yields glucose. (C) The crystal structure of lactate dehydrogenase. (D) Lactate dehydrogenase catalyzes the inter-conversion of pyruvate and lactate.

stasis biomarkers salivary amylase and lactate dehydrogenase. To test the hypothesis, the effects of THSG on the sleep time of mice were explored here. For the selection of experimental animals, the functions of $P$. multiflorum have been widely reported in Kunming mice, ${ }^{18,19)}$ and thus the mice were used for testing the effects of the THSG on sleep.

\section{Experimental Procedure}

Animals Kunming mice (three-week old) were purchased from the Center of Laboratory Animal of the Second Military Medical University (Shanghai, China). All mice were kept under $12 \mathrm{~h}$ light $/ 12 \mathrm{~h}$ dark at $22-23^{\circ} \mathrm{C}$ with relative $60 \%$ humidity and were given a diet as Table 1 showed. All the animal care and handling procedures were approved by the Institutional Animal Care and Use Committee of The Affiliated Hospital to Changchun University of Chinese Medicine.

The Purification of THSG Acetonitrile was of HPLCgrade (Merck, Darmstadt, Germany). Samples of the root of P. multiflorum were purchased from the Department of Pharmacognosy, School of Pharmacy, Second Military Medical University (Shanghai, China). P. multiflorum was identified and confirmed by the botanist Bo Liu from the Department of Pharmacognosy in the university. Dried roots of P. multiflorum were comminuted (60 mesh) and sample powder $(0.25 \mathrm{~g})$ was suspended in $70 \%$ methanol $(50 \mathrm{~mL})$ in a flask $(100 \mathrm{~mL})$ for $30 \mathrm{~min}$, which was weighed again. The flask was then sealed, shaken for a while, and extracted by ultrasonication at room temperature for $30 \mathrm{~min}$. After cooling, methanol was added to the flask to restore the original weight. After a while the supernatant liquid (approx. $10 \mathrm{~mL}$ ) was filtered and the filtrate was transferred to a volumetric flask $(10 \mathrm{~mL})$. A $10-\mu \mathrm{L}$ sample was injected for HPLC analysis. HPLC was performed with an Agilent (MA, U.S.A.) 1200 liquid chromatograph according to a previous report. ${ }^{20}$ THSG was purified with preparative HPLC (Waters, U.S.A.) Prep LC system 4000
Table 1. Compositions of the Diets Used in the Present Study

\begin{tabular}{|c|c|}
\hline \multicolumn{2}{|c|}{$\mathrm{Wt} \%$} \\
\hline Casein & $24.1 \pm 2.8$ \\
\hline Corn starch & $45.9 \pm 4.7$ \\
\hline Sucrose & $5.2 \pm 0.6$ \\
\hline Maltodextrin & $5.6 \pm 0.4$ \\
\hline Soy oil & $5.0 \pm 0.3$ \\
\hline Palm oil & - \\
\hline Lard & - \\
\hline Cellulose & $5.3 \pm 0.5$ \\
\hline Mineral mixture & $6.2 \pm 0.6$ \\
\hline Vitamin mixture & $1.2 \pm 0.2$ \\
\hline \multicolumn{2}{|c|}{$\mathrm{Kj} \%$} \\
\hline Protein & $23.4 \pm 2.1$ \\
\hline Fat & $12.5 \pm 1.5$ \\
\hline Carbohydrates & $65.0 \pm 3.6$ \\
\hline Energy content $[\mathrm{kJ} / \mathrm{g}]$ & $15.7 \pm 1.3$ \\
\hline \multicolumn{2}{|c|}{ Mean sterol contents, $\mathrm{mg} / 100 \mathrm{~g}$} \\
\hline Cholesterol & $0.51 \pm 0.08$ \\
\hline Campesterol & $1.22 \pm 0.16$ \\
\hline Stigmasterol & $2.10 \pm 0.31$ \\
\hline Sitosterol & $3.03 \pm 0.50$ \\
\hline$\Delta^{5}$-Avenasterol & $0.19 \pm 0.03$ \\
\hline Sitostanol & $1.31 \pm 0.30$ \\
\hline 24-Methylene cycloartenol & $0.32 \pm 0.02$ \\
\hline Cycloartenol & Not detectable \\
\hline Campestanol & $0.42 \pm 0.10$ \\
\hline Total sterols & $8.59 \pm 0.79$ \\
\hline
\end{tabular}

Note: The batches were analyzed in triplicate.

series with a symmetry prep C18, 7 um $(19 \times 300 \mathrm{~mm}$ i.d.) column with a flow rate of $19 \mathrm{~mL} / \mathrm{min}$. Water-phosphoric acid $(100: 0.3, \mathrm{v} / \mathrm{v})$ as solvent $\mathrm{A}$ and acetonitrile-water-phosphoric 
acid $(80: 20: 0.3, \mathrm{v} / \mathrm{v})$ as solvent $\mathrm{B}$ were used for gradient elution in $0-5$ min. $^{21)}$

THSG Treatment in Kunming Mice Sixty mice were divided into five groups: control group, THSG groups (10, 20, 40 and $80 \mathrm{mg} / \mathrm{kg} / \mathrm{d})$. The mice from THSG groups were injected intravenously with different dosages of THSG for successive $8 \mathrm{~d}$. To examine the sleep duration, a computer-controlled automated sleep-wake detection system was implemented. Electroencephalogram (EEG) and electromyogram (EMG) electrodes were implanted under deep pentobarbital sodium anesthesia $(65-75 \mathrm{mg} / \mathrm{kg})$. Two gold-plated screws served as EEG electrodes and were screwed through the skull over the right cerebral hemisphere (frontal: $1.7 \mathrm{~mm}$ lateral to midline, $1.5 \mathrm{~mm}$ anterior to bregma; parietal: $1.7 \mathrm{~mm}$ lateral to midline, $1.0 \mathrm{~mm}$ anterior to lambda) as previously reported. ${ }^{22)}$ Two other screws were implanted at the same coordinates over the left hemisphere and were used as anchor screws. Two insulated stainless steel wires served as EMG electrodes and were inserted between two neck muscles. The electrodes, which were soldered to recording leads before implantation, and the anchor screws were cemented to the skull. The recording leads were connected to a swivel contact and animals were allowed 10-14d of recovery from surgery and habituation before the experiment. The program uses a combination of the frequency distribution of the EEG and amplitude of the nuchal EMG to determine sleep-wake state. The sleeping time were examined for successive $8 \mathrm{~d}$.

RNA Isolation from Kunming Mice Brain The animals were sacrificed on day $0,2,4,8$ under anesthesia after THSG treatment. The ventrolateral preoptic nucleus (VLPO), the intermediate nucleus of the preoptic (INP) area, has been reported to promote sleep, so INP area was chosen here. Brain tissue was removed with sterile scissors, and INP area was selected and snap-frozen in liquid nitrogen (LN) and then transferred to a $-80^{\circ} \mathrm{C}$ freezer. The sample was placed in an ribonuclease (RNase)-free pestle and bathed in LN, and then ground to fine powder in $\mathrm{LN}$ for $10 \mathrm{~min}$ with multiple changes of LN. Tissue for RNA isolation was directly placed in Trizol LS (Invitrogen) lysis for the subsequent RNA isolation. On the other hand, pretreat with proteinase $\mathrm{K}$ (the tissue was incubated in $50 \mu \mathrm{g} / \mathrm{mL}$ proteinase $\mathrm{K}$ at $65^{\circ} \mathrm{C}$ for $30 \mathrm{~min}$ ) combining with other methods was essential for the successful RNA isolation.

RT-PCR and Real Time Quantitative (q)RT-PCR The level of salivary alpha amylase is closely associated with serum alpha amylase, ${ }^{23)}$ and salivary alpha amylase will affect amylase level in brain. On the other hand, LDH occurs in brain tissue. Therefore, the levels of alpha amylase and LDH from brain tissues INP area were measured by using real-time qRT-PCR on the day of sacrifice (day 0, 2, 4 and 8) under anesthesia. qRT-PCR was performed using SYBR green I master mix (Light-Cycler 480, Roche R\&D Center (China) Ltd., Shanghai, China). RNA was isolated from the brain tissues of the Kunming mice treated with different dosages of THSG, followed by cDNA synthesis and data analysis as described previously. ${ }^{24)}$ The primers for real time qPCR were: LDH, 5'-GAT ATC TTA ACT TAT GTA GC-3' and 5'-CAC TGC TCA GGA TCT TTA TC-3'; Amylase, 5'-CTC ATT GAC ATT GGT GTT GC-3' and 5'-GGA TGA GCA AGC ATA AAT CC-3'; glyceraldehyde-3-phosphate dehydrogenase (GAPDH), 5'-CCC TTC ATT GAC CTC AAC TAC-3' and 5'-CCACCT TCT TGA TGT
CATCAT-3'. GAPDH was used as an internal control.

Western Blot Analysis Protein levels of alpha amylase and LDH from brain tissues INP area were measured by using Western blot on the day of sacrifice (day 0,2, 4 and 8) under anesthesia. LDH antibody and amylase antibody was purchased from Shengshizhongfang BioSci\&Tech. Ltd., Co. (Beijing, China). $\beta$-Actin antibody was purchased from Abcam (Beijing, China). Brain tissues INP area were obtained from control group and THSG groups with prior approval from the Institutional Review Board. A total of 60 tissue pieces from the mice treated with different dosages of THSG were prepared. All tissues were homogenized in RIPA buffer ( $150 \mathrm{~mm}$ Sodium chloride, 1\% NP-40, 0.5\% sodium deoxycholate, $0.1 \%$ sodium dodecyl sulfate (SDS), $50 \mathrm{~mm}$ Tris- $\mathrm{HCl}(\mathrm{pH}$ 8.0)) and protease inhibitor Complete Mini (Roche R\&D Center (China) Ltd., Shanghai, China) was added. Proteins were separated by SDS-polyacrylamide gel electrophoresis (PAGE) and transferred to a polyvinylidene difluoride membrane (Millipore, Billerica, MA, U.S.A.). The membranes were blocked with 5\% skim milk in TBST (10 mm Tris ( $\mathrm{pH} 7.5)$ and $100 \mathrm{~mm}$ $\mathrm{NaCl}$ ) and incubated with primary antibodies in TBST with $0.5 \%$ skim milk overnight at $4^{\circ} \mathrm{C}$. The membrane was treated with primary antibodies and peroxidase-conjugated secondary antibodies $(1: 3000)$ (Amersham Biosciences, Piscataway, NJ, U.S.A.). Immunoreactive bands were visualized by chemiluminescence.

Measurement of the Activity of Salivary Alpha Amylase The saliva was collected according to a previous report. ${ }^{25}$ ) Saliva was collected on the day of sacrifice (day 0, 2, 4 and 8) and saliva secretion was stimulated in anesthetized mice using subcutaneous pilocarpine injection $(0.5 \mathrm{mg} / \mathrm{kg}$, Sigma). Saliva was collected in a pre-weighed $0.5 \mathrm{~mL}$ micro-centrifuge tube by using a hematocrit tube (Drum-mond Scientific, Broomall, PA, U.S.A.) in the oral cavity for half hour. The salivary amylase activity was measured by amylase assay kit (ab102523) (Abcam (Shanghai) branch, Shanghai, China).

Measurement of Lactate, LDH and Glucose LDH in brain tissues INP area was measured using LDH activity assay kit (MAK066, Sigma). Twenty-five microliters of blood sample was taken from each mouse via tail vein and placed in phosphate buffered saline (PBS) buffer ( $\mathrm{pH} 8.0$ ) with $1 \mathrm{~mm}$ ethylenediaminetetraacetic acid (EDTA). Blood lactate concentrations were measured by L-lactate assay kit (ab65331, Abcam). Blood glucose concentrations were measure by a glucose kit (ab65333, Abacam).

Statistical Analysis The association between the concentrations of THSG and the protein levels of LDH or amylase was compared by one-way ANOVA followed by the post hoc test of Fisher's protected least significant difference. We used Spearman's rank correlation coefficient to identify the strength of correlation between the protein levels of LDH or amylase, and sleep duration, and between the protein levels of LDH or amylase and the concentrations of lactate or glucose. The online software computes the Spearman Rank Correlation and the two-sided $p$-value.

\section{Results}

The Purification of THSG $P$. multiflorum is a traditional Chinese herb that has been widely used as a tonic and anti-aging agent in the Orient since thousand years ago. $P$. multiflorum consists of anthraquinone, stilbene, phospholipid, 

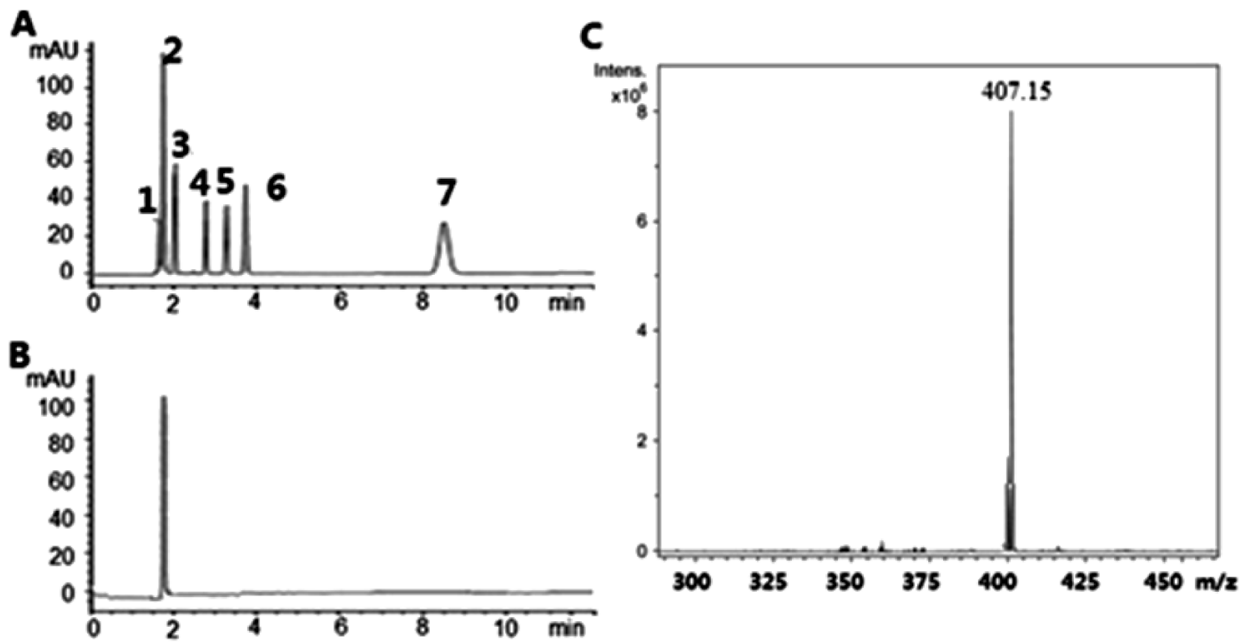

Fig. 2. The Purification of THSG

(A) Analytical HPLC analysis showed seven components in the extracts of P. multiflorum. (B) THSG was purified by preparative HPLC using a Waters C18 reversedphase cartridge with $0.1 \%$ TFA and $\mathrm{CH}_{3} \mathrm{CN}$ as mobile phase. (C) The molecular weight of THSG was verified with ESI MASS spectrometry. Only peak $407.15\left([\mathrm{M}+\mathrm{H}]^{+}\right)$ was observed (expected mass: 406.38 Da, calculated mass: 406.15 Da).

and other compounds (Fig. 2A). The components and chemical structure were consisted with previous reported. ${ }^{7)}$ THSG $\left(2,3,5,4^{\prime}\right.$-tetrahydroxystilbene-2- $O-\beta$-D-glucoside) (Fig. $\left.2 \mathrm{~B}\right)$ is one of the main components that can be extracted from the root of $P$. multiflorum. ${ }^{26}$ ) A growing body of evidence has shown that THSG exhibits significant antioxidant activities both in vitro and in vivo. ${ }^{27,28)}$ To test the function of THSG, the purification of THSG becomes very important. The analytical HPLC analysis showed that there are seven components from the extracts of $P$. multiflorum. Each component was further purified by preparative HPLC. From the results of electrospray ionization (ESI) MASS, the second component was THSG (Figs. 1B, C). The observed mass is $406.15 \mathrm{Da}$, which is consistent with the theoretical mass 406.38 .

The Effects of THSG on Sleep Patterns of Mice The sleep duration can be measured by autography, which provides objective assessments of sleep patterns. ${ }^{29)}$ Normally, Kunming mice can sleep averagely $13 \pm 2 \mathrm{~h}$ per day without THSG treatment. Electroencephalogram (EEG) and electromyogram (EMG) showed that THSG could extend the sleep time of mice and the time reached up to $15 \pm 2 \mathrm{~h}$ per day when $80 \mathrm{mg} / \mathrm{kg}$ THSG was injected each day $(p<0.01 v s$. the control). There are seven components from the extracts of $P$. multiflorum. The mice were treated with other six components in the same way. The sleep time of the mice did not change ( $p>0.05$ vs. the control). The total amount of wake time during the dark period was almost similar compared with the mice without THSG treatment (Fig. 3). This reduction in total wake amount occurred in dark period (Fig. 3A). In addition, THSG treatment $(80 \mathrm{mg} / \mathrm{kg} / \mathrm{d})$ increased NREM sleep time and reduced REM sleep time ( $p<0.05$, Figs. 3B, C). Furthermore, the sleep profile was similar between the mice without any treatment and treated with the extracts of $P$. multiflorum without THSG ( $p>0.05$, Figs. 3A-C).

The Effects of THSG on mRNA Levels of LDH and Amylase To explore the molecular mechanism of THSG causing the change of the sleep time, LDH and saliva alpha amylase were chosen. First, the mRNA levels of both molecules were investigated. The method quantitative (real-time) qRT-PCR analysis was performed here. Before qRT-PCR analysis, the integrity of RNA is often problematic in RNA isolation. Single mouse brains contain low levels of the interested gene mRNA, which are beyond detectable. Therefore, it was necessary to find the optimal methods to isolate sufficient RNA suitable for successful amplification. We tested four extraction methods, all of which yielded enough RNA, varying in integration and concentration. Integration could be increased by the addition of SDS and other denaturing chemicals. Methods that involved heat treatment yielded the RNA with higher concentration than that obtained from the methods without heat treatment. The proteinase $\mathrm{K}$, liquid nitrogen and guanidinium chloride pretreatment produced high integrated RNA from mice brains. Thus, in terms of RNA quality, the pretreatment is necessary to prevent degradation of the RNA.

qRT-PCR analysis showed the mRNA levels of LDH and amylase decreased after THSG treatment. The inhibitory effects of THSG for LDH and amylase were in a dose-dependent way. The mRNA levels of LDH and amylase were inhibited significantly when $80 \mathrm{mg} / \mathrm{kg}$ of THSG was used (Figs. 4A, B).

The Effects of THSG on the Protein Levels of LDH and Amylase Considering the different clinical situations of sleep, the protein levels of LDH and amylase were measured via Western blot. The analysis showed that the protein level of LDH was reduced after THSG treatment ( $p<0.05$ via control). The expression of LDH decreased obviously $(p<0.001$ via control) when $80 \mathrm{mg} / \mathrm{kg}$ of THSG was used (Fig. $5 \mathrm{~A}$ ). The results suggested that the expression of LDH was down-regulated by THSG. Similarly, the protein expression of amylase decreased after THSG treatment $(p<0.05$ via control). The expression of amylase decreased significantly $(p<0.001$ via control) when $80 \mathrm{mg} / \mathrm{kg}$ of THSG was used (Fig. $5 \mathrm{~B}$ ).

The Effects of the Protein Levels of $\mathrm{LDH}$ and Amylase on Sleep Duration Spearman's rank correlation coefficient showed that relative protein levels of LDH were increased while the sleep duration was reduced. Relative protein levels of LDH are negatively associated with sleep duration $(p<0.05$, Fig. 6A). Similarly, relative protein levels of amylase were increased while the sleep duration was reduced. Relative protein levels of amylase are negatively associated with sleep duration 

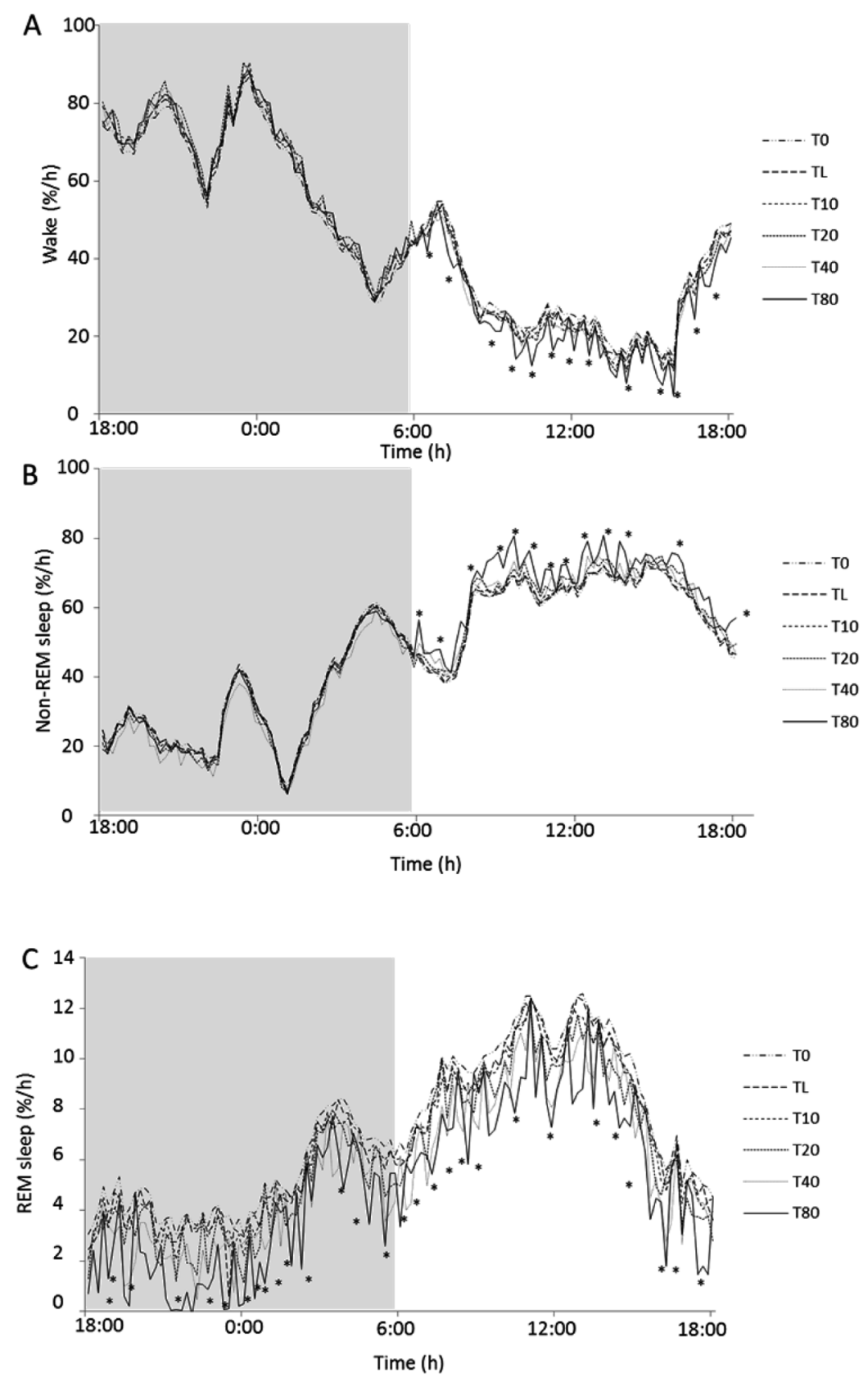

Fig. 3. The Effects of THSG from P. multiflorum on Sleep Time of Mice

T0, T10, T20, T40 and T80 stand for the mice orally administrated with THSG at the dose of $0,10,20,40$ and $80 \mathrm{mg} / \mathrm{kg} / \mathrm{d}$. TL stands for the mice treated by total extracts of $P$. multiflorum without THSG. A, The effects of THSG on wake. B, the effects of THSG on non-REM sleep. C, the effects of THSG on REM sleep. The dark period is presented as shaded area. $p<0.05$ via a control group without THSG treatment.

$(p<0.05$, Fig. 6B).

The Relation between THSG Concentration and the Activities of LDH and Amylase Figures 7A and B showed that negative relation between THSG concentration and activities of LDH and amylase. The THSG concentration was increased while the protein levels of LDH were decreased. The inverse relation between the two variables was consistent with that the results analyzed by qRT-PCR and Western blot. The test of spearman's rank correlation coefficient showed the negative relation between the THSG concentration and LDH activity $(R h o=-0.68)$. All these results suggested that the negative relation between THSG concentration and the LDH activity. The results were accordant with previous report that pretreatment with THSG markedly attenuated the release of LDH. ${ }^{26)}$ Simi- larly, the negative relation between the THSG concentration and activity of saliva amylase was also found $(\mathrm{Rho}=-0.72)$. The activity amylase was reducing when the THSG concentration was increased. The negative relation between the two variables was also consistent with above that analyzed by qRT-PCR and Western blot. However, the mechanism for THSG down-regulating the protein levels of LDH remains unknown. For the relation between THSG concentration and the activity of salivary alpha amylase, the reason remains unknown either.

The Relation between LDH Activity and Lactate Level The lactate level was associated with LDH activities. The lactate level was increasing when the activity of LDH was increased (Fig. 8, $p<0.05$ ). According to previous report, high- 
A

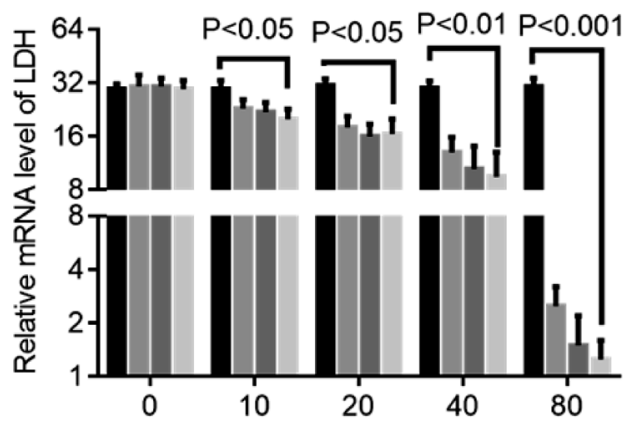

The concentration of THSG $(\mathrm{mg} / \mathrm{kg} / \mathrm{day})$

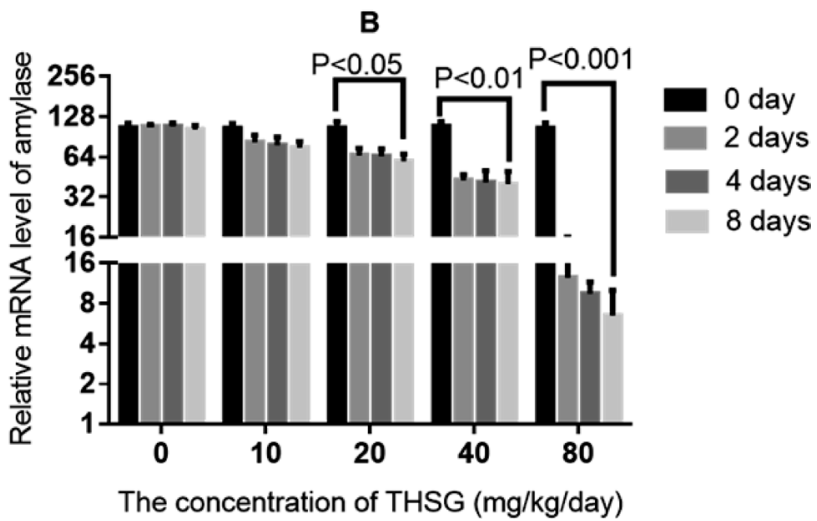

Fig. 4. The Relative mRNA Levels of LDH and Amylase in the Brain of Kuming Mice Treated with THSG or Untreated

(A) The relative mRNA levels of LDH in the brain of Kuming mice treated with THSG or untreated. (B) The relative mRNA levels of amylase in the brain of Kuming mice treated with THSG or untreated. Each bar represented the mean \pm S.D. of three independent experiments.

$\mathrm{LDH}$
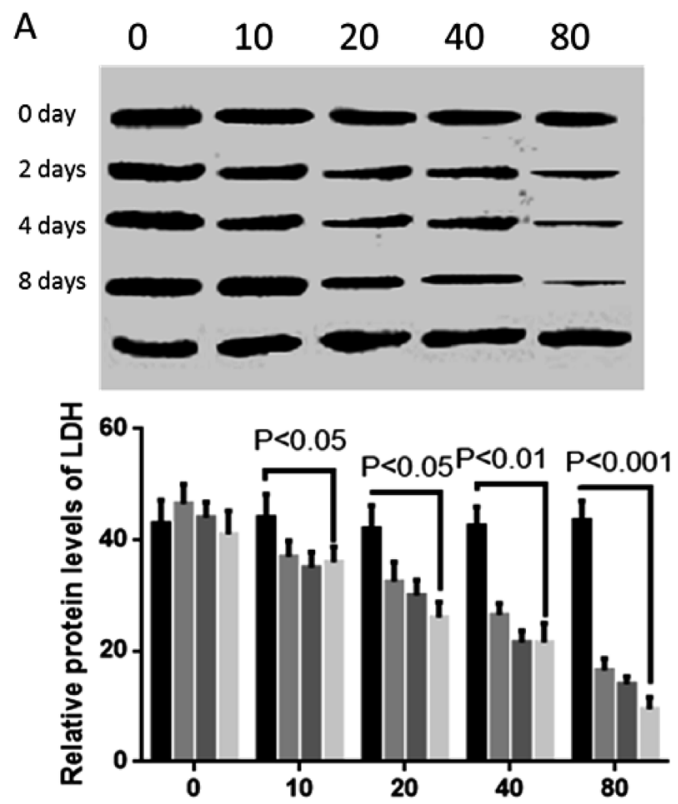

The concentration of THSG $(\mathrm{mg} / \mathrm{kg} / \mathrm{day})$
Amylase

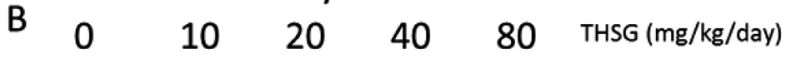
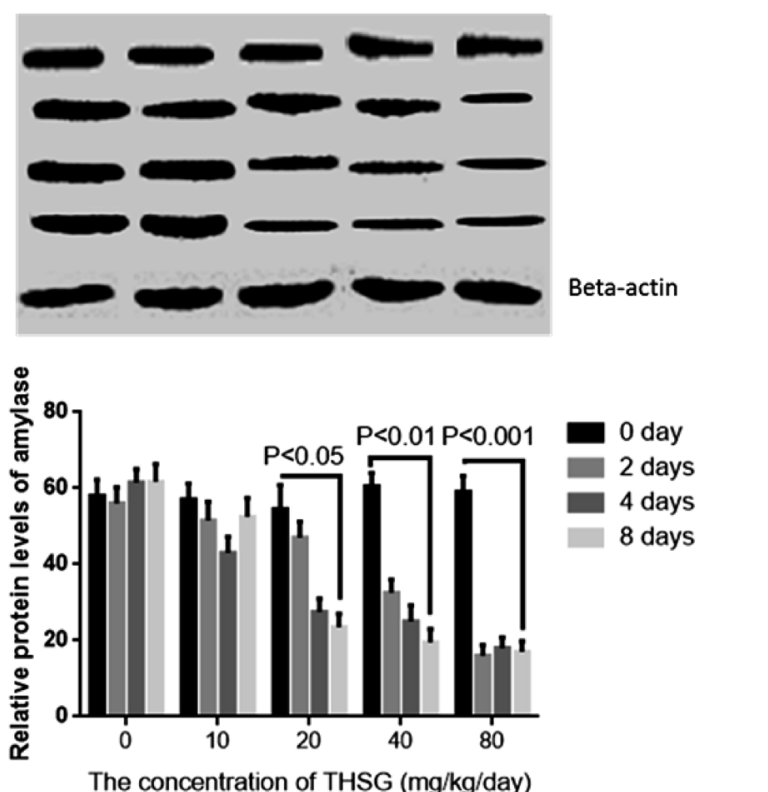

Fig. 5. Western Blot Analysis for the Relative Protein Levels of LDH and Amylase

(A) The relative protein levels of LDH change in the mice when they are treated with different dosages of THSG; (B) The relative protein levels of amylase change in the mice when they are treated with different dosages of THSG. Up panel, Western blots analysis for the protein levels of LDH and amylase. $\beta$-Actin was shown as a control. Down panel, the columns correspond to the blotting dot in up panel. Each bar represented the mean \pm S.D. of three independent experiments.
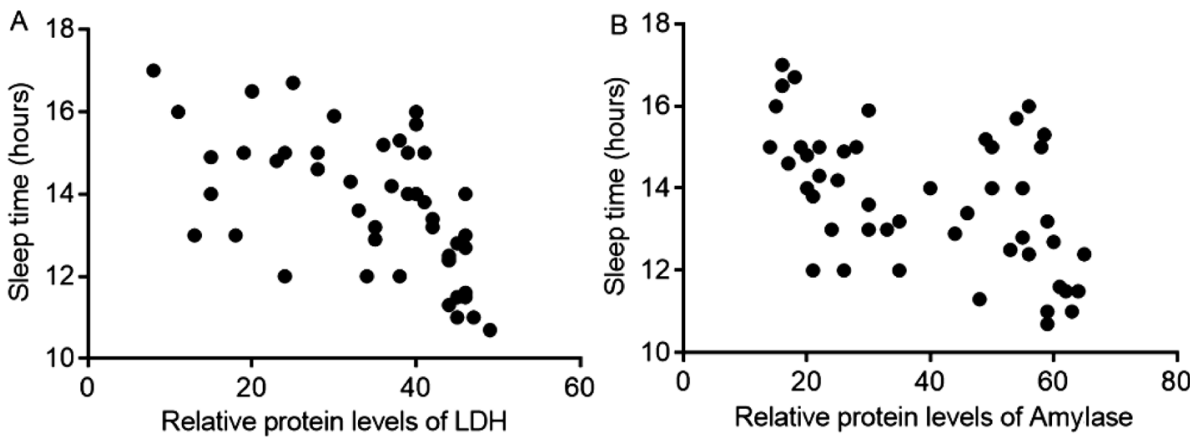

Fig. 6. Spearman's Rank Correlation Coefficient for the Effects of the Protein Levels of LDH and Amylase on Sleep Duration

(A) The effects of the protein levels of LDH on sleep duration ( $p=0.001$ and Rho $=-0.71$ ). (B) The effects of the protein levels of amylase on sleep duration ( $p=0.001$ and $\mathrm{Rho}=-0.58$ ). There was a strong negative relation between two variables if Rho falls between -0.5 and -1 . 
A

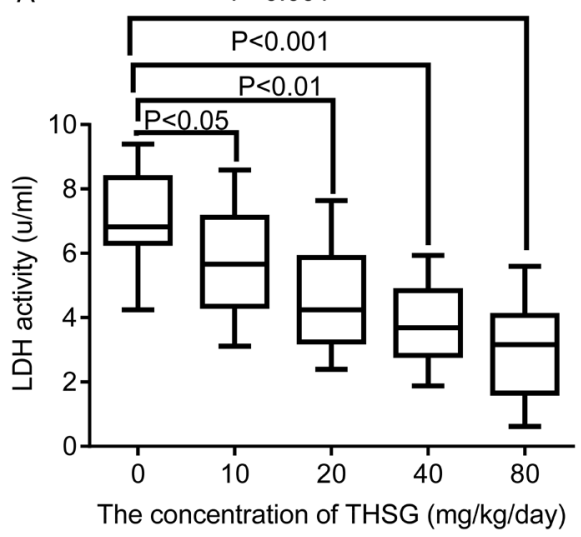

B

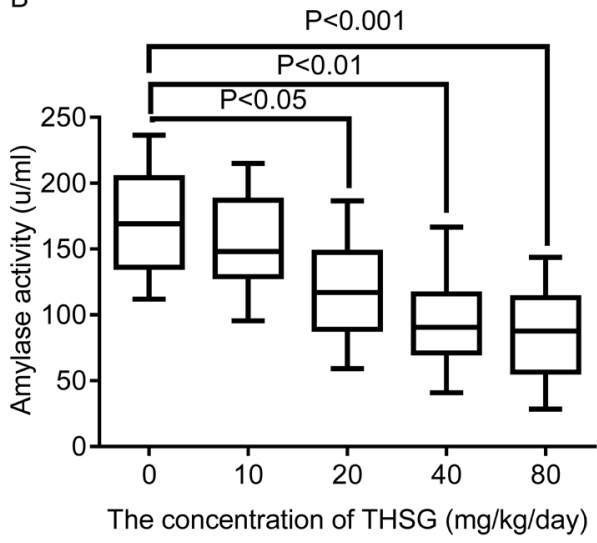

Fig. 7. The Relation between THSG Concentration and Activities of LDH and Amylase

(A) The relation between THSG Concentration and LDH activity. (B) The relation between the THSG concentration and amylase activity.

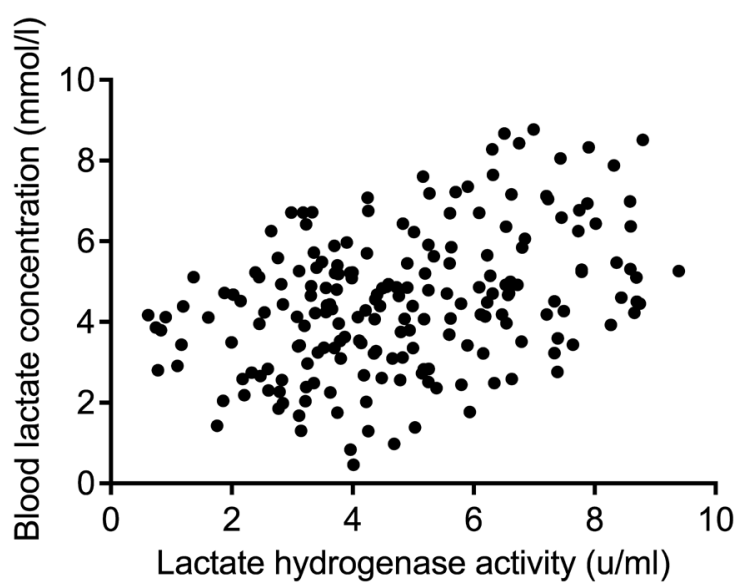

Fig. 8. The Relation between LDH Activity and Lactate Level

$p=0.001$ and $\mathrm{Rho}=0.74$. There was a strong relation between two variables if Rho falls between 0.5 and 1 .

level of lactate will keep awaking situation. ${ }^{15)}$ THSG reduced lactate level by inhibiting $\mathrm{LDH}$ activity and would promote sleep homeostasis.

The Relation between Alpha Amylase Activity and Glucose Level The glucose level was associated with salivary alpha amylase activities. The glucose level was increased when the activity of amylase was increased (Fig. 9, $p<0.05$ ). According to previous report, poor regulation of glucose will result in sleep impairment. ${ }^{30)}$ Short sleep durations have been reported to be related with dysregulation of glucose in the patients with gestational diabetes. Poor blood glucose control is closely associated with sleep disorders. ${ }^{30)}$ Furthermore, poor glucose control is also related with Obstructive Sleep Apnea Syndrome, insulin resistance, type 2 diabetes mellitus and cardiovascular disease. ${ }^{31)}$ THSG reduced glucose concentration by inhibiting amylase activity and would promote sleep homeostasis.

\section{Discussion}

Sleep disorder is still threatening public health and difficult to be treated worldwide. ${ }^{32)}$ Here, we found that THSG could affect the levels of LDH and amylase, which were the sleep homeostasis biomarkers related with sleep time. THSG affects the sleep duration of mice by down-regulating the

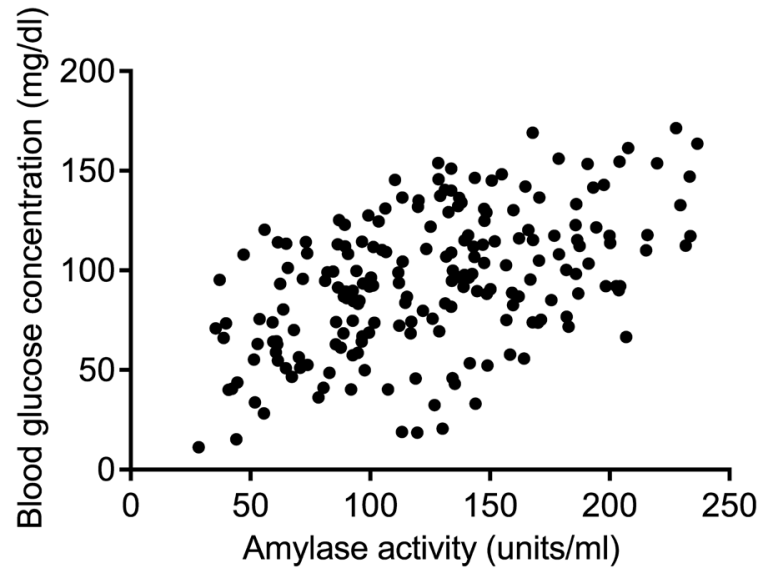

Fig. 9. The Relation between Amylase Activity and Glucose Level

$p=0.001$ and $\mathrm{Rho}=0.78$. There was a strong relation between two variables if Rho falls between 0.5 and 1 .

protein levels of LDH and salivary alpha amylase. The study may provide useful information for exploring the function of THSG for preventing insomnia. However, more frequent use of Shouwuteng (the stems of P. multiflorum) involved practice of $\mathrm{CHM}^{33)}$ and that this frequent use is as an addition to a complex formula. ${ }^{34)}$ These components seem too complex and the molecular mechanism cannot be explored with present technology. On the other hand, "Shouwuteng," the most commonly prescribed Chinese herb for the subjects with insomnia, has been reported to be with anti-inflammatory activity, ${ }^{35)}$ an anti-atherosclerogenic effect ${ }^{36)}$ and exhibits neuroprotective effects in animal studies. ${ }^{37)}$ The current study was done with P. multiflorum roots, with one main component, THSG. The stems of $P$. multiflorum with different components, are frequently used in treating insomnia. ${ }^{5)}$ Here, we only considered the function of THSG for prevening insomnia. According to previous report, the amounts of THSG in root was almost 3 times more than that in stem $(1.5 \mathrm{mg} / \mathrm{g}$ THSG in root and $0.4 \mathrm{mg} / \mathrm{g}$ THSG in stem), ${ }^{38)}$ so the root of $P$. multiflorum was considered here. The safety and efficacy of THSG remains uncertain. Although current data suggests the use of some herbal treatments in insomnia may be efficacious, further laboratory and clinical studies are required.

THSG can change the sleep profile by reducing wake and 
REM periods and increasing NREM period when its concentration is more than $80 \mathrm{mg} / \mathrm{kg}$ (Fig. 3). THSG can reduce the activities of amylase and LDH. Meanwhile, the levels of amylase and LDH are negatively associated with sleep duration (Fig. 6). Thus, THSG improves sleep situation by affecting the levels of amylase and LDH.

THSG changes LDH, salivary alpha amylase, lactate and glucose, so we are wondering whether benzodiazepines or barbiturates affect these factors. Benzodiazepines are used to treat symptoms of sleep disorder. ${ }^{39)}$ The drugs have been early reported to inhibit lactate dehydrogenase too. ${ }^{40)}$ Another report shows that benzodiazepines inhibit amylase release from rat parotidacinar cells. ${ }^{41)}$ A barbiturate is a kind of medicine as a nervous depressant, and can induce sleep. ${ }^{42)}$ The drug has been found to inhibit the activity of amylase. ${ }^{43)}$

Frankly, there are some drawbacks for the study. Firstly, it is obvious this compound cannot represent the long-documented efficacy and safety, although the safety is only tested in animals. ${ }^{44}$ The current paper is still not certain for the safety and efficacy of THSG. There is no effort to directly link the tested THSG to actual clinical use of herbal treatments for insomnia, no safety testing performed, and no data provided in the support of efficacy. Secondly, based on the amounts of THSG in the roots, ${ }^{38)}$ the implication is that the THSG used in the study is at a substantially higher dose than consumed by the patients who receive the medicine in decoctions. The amounts are not a problem when the medicine is only used for the mice test, but it seems too many for human use. The problem may be solved by combining with other medicine. Thirdly, all these subjects of amylase activity and mechanisms of sleep previously discussed, and prior findings have not been tested in in vivo. It still cannot be developed as a standard practice. All previous work for this purpose has been performed in fruit flies, mice, and rats respectively, but not humans. ${ }^{12,13,15,16)}$ Thus, system test in animals or clinical trials seem to have become very important in future.

\section{Conclusion}

The stringent relationship between the concentrations of THSG and the protein levels of LDH or amylase was firstly reported here. The level of LDH and amylase is down-regulated by THSG. The levels of lactate and blood glucose concentration are closely associated with the activities of $\mathrm{LDH}$ and amylase, and affect sleep homeostasis. LDH and amylase are potentialy combined sleep homeostasis biomarkers related with the sleep. The study may provide useful information for exploring the function of THSG for preventing insomnia.

Conflict of Interest The authors declare no conflict of interest.

\section{References}

1) Tamura N., Tanaka H., J. Behav. Med., 40, 659-668 (2017).

2) Tachikawa R., Minami T., Matsumoto T., Murase K., Tanizawa K., Inouchi M., Oga T., Chin K., Ann. Am. Thorac. Soc., 14, 986-993 (2017).

3) Vgontzas A. N., Liao D., Bixler E. O., Chrousos G. P., Vela-Bueno A., Sleep, 32, 491-497 (2009).

4) Vgontzas A. N., Liao D., Pejovic S., Calhoun S., Karataraki M., Basta M., Fernández-Mendoza J., Bixler E. O., Sleep, 33, 1159-1164 (2010).
5) Wing Y. K., Hong Kong Med. J., 7, 392-402 (2001).

6) Chen F.-P., Jong M.-S., Chen Y.-C., Kung Y.-Y., Chen T.-J., Chen F.-J., Hwang S.-J., Evid. Based Complement. Alternat. Med., 2011, 9 (2010).

7) Wei Y. J., Li S. L., Li P., Biomed. Chromatogr., 21, 1-9 (2007)

8) Zhang L., Xing Y., Ye C.-F., Ai H.-X., Wei H.-F., Li L., Behav. Brain Res., 173, 246-254 (2006).

9) Hou Y., Yang Q., Zhou L., Du X., Li M., Yuan M., Zhou Z., Li Z., Can. J. Physiol. Pharmacol., 89, 801-809 (2011).

10) Seugnet L., Boero J., Gottschalk L., Duntley S. P., Shaw P. J., Proc. Natl. Acad. Sci. U.S.A., 103, 19913-19918 (2006).

11) Xu X., Wang L., Zhang Y., Su T., Chen L., Zhang Y., Ma W., Xie Y., Wang T., Yang F., He L., Wang W., Fu X., Hao H., Ma Y., Sleep Biol. Rhythms, 14, 321-328 (2016).

12) Cirelli C., LaVaute T. M., Tononi G., J. Neurochem., 94, 1411-1419 (2005).

13) Stern J. E., Hestrin S., Armstrong W. E., J. Physiol., 526, 109-114 (2000).

14) Hayashida K., Suzuki M., Yonemoto N., Hori S., Tamura T., Sakurai A., Tahara Y., Nagao K., Yaguchi A., Morimura N., SOSKANTO 2012 Study Group, Crit. Care Med., 45, e559-e566 (2017).

15) Naylor E., Aillon D. V., Barrett B. S., Wilson G. S., Johnson D. A., Johnson D. A., Harmon H. P., Gabbert S., Petillo P. A., Sleep, 35, 1209-1222 (2012).

16) Dash M. B., Tononi G., Cirelli C., Sleep, 35, 909-919 (2012)

17) Gutmann I., Wahlefeld A. W., "Methods of Enzymatic Analysis," Vol. 3, ed. by Bergmeyer H. U., Academic Press, New York and London, 1975, pp. 1464-1468.

18) Wang X., Zhao L., Han T., Chen S., Wang J., Eur. J. Pharmacol., 578, 339-348 (2008).

19) Zhou X.-X., Yang Q., Xie Y.-H., Sun J.-Y., Qiu P.-C., Cao W., Wang S.-W., Phytochem. Lett., 6, 372-378 (2013).

20) Zhang F., Chen W., Sun L., Chromatographia, 67, 869-874 (2008).

21) Govindarajan R., Vijayakumar M., Shirwaikar A., Rawat A. K. S., Mehrotra S., Pushpangadan P., Acta Pharm.-Zagreb, 56, 489 (2006).

22) Franken P., Malafosse A., Tafti M., Am. J. Physiol., 275, R1127R1137 (1998).

23) Ahmadi-Motamayel F., Goodarzi M. T., Jamshidi Z., Mahdavinezhad A., Rafieian N., Brazilian Dental Science, 19, 40-46 (2016).

24) Hanning J. E., Groves I. J., Pett M. R., Coleman N., Virol. J., 10, 159 (2013).

25) Koopman F. A., Vosters J. L., Roescher N., Broekstra N., Tak P. P., Vervoordeldonk M. J., Oral Dis., 21, 858-865 (2015).

26) Qin R., Li X., Li G., Tao L., Li Y., Sun J., Kang X., Chen J., Toxicol. Lett., 202, 1-7 (2011).

27) Zhang Y., Ma K.-Y., Dong R., Journal of Jining Medical University, 1, 9 (2012)

28) Yu J., Xie J., Mao X.-J., Wei H., Zhao S.-L., Ma Y.-G., Li N., Zhao R.-H., Chin. J. Nat. Med., 10, 63-67 (2012).

29) Kawada T., Psychopharmacology (Berl.), 228, 509 (2013).

30) Twedt R., Bradley M., Deiseroth D., Althouse A., Facco F., Obstet. Gynecol., 126, 326-331 (2015).

31) Kallianos A., Trakada G., Papaioannou T., Nikolopouloss I., Mitrakou A., Manios E., Kostopoulos K., Kostopoulos C., Zakopoulos N., Eur. Rev. Med. Pharmacol. Sci., 17, 1932-1937 (2013).

32) Bhidayasiri R., Sringean J., Rattanachaisit W., Truong D. D., J. Neurol. Sci., 374, 32-37 (2017).

33) Hsieh S. C., Lai J. N., Lee C. F., Hu F. C., Tseng W. L., Wang J. D., Pharmacoepidemiol. Drug Saf., 17, 609-619 (2008).

34) Chen H. C., Hsieh M. T., Clin. Ther., 7, 334-337 (1985).

35) Li R. W., David Lin G., Myers S. P., Leach D. N., J. Ethnopharmacol., 85, 61-67 (2003).

36) Yang P.-Y., Almofti M. R., Lu L., Kang H., Zhang J., Li T.-J., Rui Y.-C., Sun L.-N., Chen W.-S., J. Pharmacol. Sci., 99, 294-300 (2005)

37) Li X., Matsumoto K., Murakami Y., Tezuka Y., Wu Y., Kadota S., 
Pharmacol. Biochem. Behav., 82, 345-352 (2005).

38) Shao L., Cui T.-B., Zhao S.-J., Journal of South China University of Technology, 38, 147-150 (2010).

39) Bourgeois J., Elseviers M. M., Van Bortel L., Petrovic M., Vander Stichele R. H., Drugs Aging, 31, 677-682 (2014).

40) Martini C., Lucacchini A., Ronca G., Bulletin of Molecular Biology and Medicine, 6, 1-5 (1981).

41) Okubo M., Kawaguchi M., Eur. J. Pharmacol., 359, 243-249
(1998).

42) Quadro G., Thiazole derivative and pharmaceutical compositions containing it. Google Patents, U.S. 4447419 (1984).

43) Bedetti C., Bozzini A., Silano V., Vittozzi L., Biochim. Biophys. Acta, 362, 299-307 (1974).

44) Chen J., Zhao H., Luo L., Zheng C., Wang F., Shi Q., Liu B., Wang W., Afr. J. Pharm. Pharmacol., 6, 1022-1025 (2012). 